\title{
Profile-based IN Service Provision for Third Generation Mobile Communication Networks
}

\author{
Hoang Nguyen-Minh, Harmen R. van As \\ Vienna University of Technology, Institute of Communication Networks \\ Favoritenstrasse 9/388, A-1040, Vienna, Austria \\ Tel.: + 43-1-58801-38815, Fax: + 43-1-58801-38898 \\ Email: Hoang.Nguyen-Minh@tuwien.ac.at
}

Abstract: The number of mobile users requiring high-quality and mobility-related services is increasing ever so rapidly. An important issue is efficient management of service provisioning, locating mobile users as well as operating network service databases. In this paper, we introduce a system model that integrates the intelligent network (IN) and mobile communication networks, thus providing fast, flexible IN service design, creation, and provision independent of the infrastructure of the diverse communication networks. This paper also examines a profile-based replication strategy, a user profile replication (UPR) that can reduce the update costs. We also present a method to distribute network databases and service control management of the VLRs and MSC/SSPs by taking into account user mobility behaviour. A most frequent location area (MFLA) is introduced which also reduces the number of location updates in such a way that the change of the location information and the service profile database are managed by the VLR/MFLA of the user instead by the HLR/SCP. With the MFLA concept, the currently centralized database and the service control management becomes distributed. This reduces the communication (signaling messages) and computational cost (database accesses) as compared to the distributed VLR scheme and a reference scheme for UMTS/IMT-2000 regarding IN service provision and location management.

Keywords: IMT-2000, UMTS, Intelligent Network (IN), HLR, VLR, location update, mobility management.

The original version of this chapter was revised: The copyright line was incorrect. This has been corrected. The Erratum to this chapter is available at DOI: 10.1007/978-0-387-35522-1_37 


\section{INTRODUCTION}

The third generation mobile communication systems (UMTS/IMT-2000) are designed to provide global operation and an enhanced set of services capabilities while at the same time improving network performance significantly. These networks will evolve from the existing wireless and wired networks by adding the necessary capabilities to support intelligent and mobility-related services and features. For that propose, the evolution will be based on increased IN capabilities for rapid introduction of services, efficient service control and provision, incorporating database and signaling capabilities necessary to support terminal, personal, and service mobility functions.

Many research works have been carried out to develop IN concepts for third-generation mobile communication networks. In [1], a network model to integrate the common functionalities of the mobile network and a method to provide the IN service efficiently have been proposed. This method downloads the IN service profile to the Visitor Location Register (VLR) registered by the mobile terminal (MT) during location registration. It also provides the IN service to the VLR when an IN service call arrives from the MT. In this paper, this scheme is referred to as the Lee's scheme. In [2], a first step towards a user-profile management and a virtual home environment (VHE) by employing IN capabilities set 3 (CS-3) has been proposed. A vertical architecture with full independence of the user-profile database and the service management can be used to facilitate the provision of multiple services, allowing services to be active in parallel.

In this paper, an integration of the IN and mobile communication networks is studied. We also propose a profile-based replication that can be applied to distribute service provision and location management for future mobile communications. A new location strategy called user-profile replication (UPR) is proposed. UPR reduces the communication (signaling messages) and computational costs (database accesses) as compared to Lee's scheme [1] and a reference scheme used for UMTS/IMT-2000 [5,6]. A most frequent location area (MFLA) is then introduced which also reduces the number of location update messages between the Home Location Register (HLR) and the Visitor Location Register (VLR) in such a way that the location information change and service profile downloading are managed by the MFLA of the user instead of the HLR and the Service Control Point (SCP). The UMTS/IMT-2000 scheme is considered as reference architecture for comparison. We give the protocols of location updating/registration and $\mathbb{N}$ service call procedures. We compare the three algorithms and show the effectiveness of the UPR scheme by an analytical method.

The rest of the paper is organized as follows. In Sections 2 and 3, we describe the IN capabilities set (CS) and the integrated system model for mo- 
bile communication systems. In Section 4, we introduce the procedures for location update and IN service call setup of those schemes. We present an analysis and performance comparison of these algorithms in Section 5. Finally, some concluding remarks are given in Section 6. A list of abbreviations is given at the end of the paper.

\section{IN CAPABILITIES SETS (IN CS) IN MOBILE NETWORKS}

UMTS/IMT-2000 are third-generation mobile communication systems that aim to support global service portability with a QoS that is at least comparable to that offered by the wired networks in various wired and wireless environments. UMTS/IMT-2000 should take care of the interest of developing and deploying personal communication services (PCSs) in the sense that it could use functional model on IN functional entities (FEs) to develop new services in a modular way.

IN is a high-level network architecture that facilitates and speeds up service implementation and provisioning in a cost-effective manner. In addition, IN can be applied to several types of networks such as PSTN, ISDN, and mobile networks. The IN architecture based on CS-1/2/3 capabilities can be used to support mobile communication networks and the interest of integrating them in IMT-2000/UMTS has increased [2, 5, 6]. ITU-T has proposed the IN capability sets (CS) such as CS-1, CS-2, and CS-3 [3, 4]. CS-1 advocates a centralised architecture in the sense that it does not support the distribution of control and data storage functionalities. CS-1 specifies the IN services at the wired network level and generally supports only personal mobility. In CS-2, not only personal and terminal mobility is provided, but also service mobility in mobile networks. CS-3 is advised to provide integrated services of wired and wireless networks, as well as the first set of capabilities for service cohabitation.

ITU-T also specifies the IN Conceptual Model (INCM) that is the framework to describe and design the $\mathbb{I N}$ architecture. This model provides four views of telecommunication networks through which the services could be modelled. It comprises the Service Plane, the Global Functional Plane, the Distributed Functional Plane, and the Physical Plane. These four planes show the capability of IN from each different side [3, 4]. 


\section{SYSTEM MODEL FOR INTEGRATING OF IN AND MOBILE NETWORKS}

In this section, we analyse the integrated model and study how IN services are provisioned in the third generation mobile communication networks such as UMTS/IMT-2000. The proposed system model for third mobile communication networks based on IN is shown in Figure 1. In this configuration, MSC/SSPs (service switching points in the mobile switching centers) and the service control points (SCPs) are interconnected over a common channel-signaling network (SS7 network).

In IN, the SSP recognizes the request for the IN service and then sends the query to the SCP, which processes the IN service profile and returns it to the SSP. In mobile communication networks, the Home Location Register (HLR) and the Visitor Location Registers (VLRs) have been used to manage the location of the MT and the subscription profile of the users. Thus, an SCP constitutes a distributed transaction system in which functions are distributed among the service control modules (SCMs), the service data module (SDM), and the HLR. The HLR in the model only stores the home user profiles and their current location. The SDM can be divided into many modules, which store a common set of data for all the services supported by that domain. With this integration model it is possible to efficiently implement various services as well as to ensure mobility. This model also supports the separation of the mobility-related data managed at the transport level from the user-related service data managed at the service level.

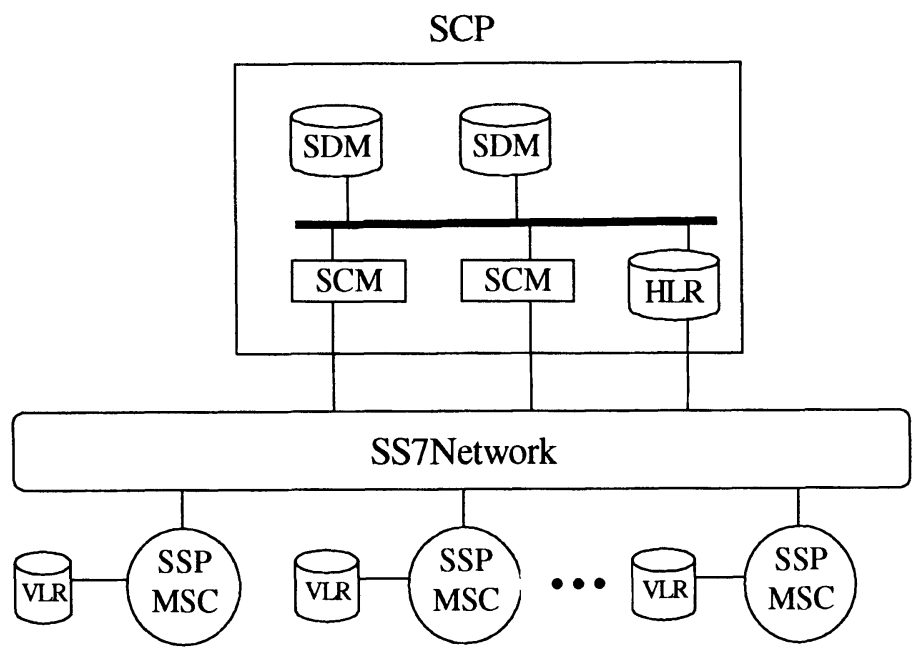

Figure 1. Reference architecture 


\subsection{Lee's Scheme}

Lee et al. have proposed a distributed VLRs scheme [1], which can reduce the signaling costs and the load of the SCP. In this strategy, the SDM is distributed to the VLR where the user is currently located. User and service profiles are downloaded to the VLR when an MT performs the location registration. Another aim of the VLR is to response to $\mathbb{I N}$ service requests from the corresponding MT during the call setup procedure. The MSC/SSP recognizes the IN calls of the MT and sends the IN service request to the VLR instead of the SCP.

\subsection{User profile replication (UPR) with MFLA strategy}

\section{A. Strategy Definition}

In the UPR, the system handles a per-user mobility profile recording his most probable mobility patterns, $\left\{\left(A_{1}, p_{\mathrm{A} 1}\right),\left(A_{2}, p_{\mathrm{A} 2}\right) \ldots\left(A_{\mathrm{k}}, p_{\mathrm{Ak}}\right)\right\}$, whereby $p_{\mathrm{Ai}}$ denotes the probability that an MT is in location $A_{\mathrm{i}}$, and $p_{\mathrm{A} 1}>p_{\mathrm{A} 2}>\ldots>$ $p_{\text {Ak. }}$. The probability $\kappa$ to find an MT in $\left\{A_{i}\right\}_{i=1}^{k}$, whereby $k$ is number of location areas, is given by

$$
\kappa=\sum_{i=1}^{k} p_{A i}
$$

The profile for each user is stored in the SDM/SCP and the VLRs by $\left\{A_{i}\right\}_{i=1}^{k}=\left\{A_{1}, A_{2}, \ldots, A_{k}\right\}$. Each user must store an ordered set of locations $\left\{A_{i}\right\}$ considered as a location area for him in its registered MT. The first entry in $\left\{A_{i}\right\}, A_{l}$, is called the most frequent location area (MFLA). Within $\left\{A_{i}\right\}$, the MT does not generate a location update. For incoming calls, the MT has to be paged in all the locations in the $\left\{A_{i}\right\}$. When the MT moves outside $\left\{A_{i}\right\}$, it has to register the new location to the VLR/MFLA instead of the HLR/SCP. If an IN service is requested from an MT, the IN service profile and the service-related database can be provided locally by the replicated VLR where the MT is currently located. It has then not to be provided by the SCP.

This scheme can reduce the number of unnecessary location updates by taking into account the user mobility patterns, but it increases paging delays, required memory, as well as profile updating cost.

\section{B. User Profile List (UPL) updating and maintaining}

Replicating user profiles can reduce the location update time. This is because the location of the called MT is more likely to be obtained by a single query from the local VLR, rather than by a high-latency remote query. However, the associated cost of replication with caching is the update cost incurred in 
processing the user profile to form the user profile list (UPL), replicating and maintaining consistent replicas to the locations where it is stored. Denote this cost as $C_{m}(k)$,whereby $k$ is the user profile size. We assume that the frequency at which the system invokes the list maintenance function is proportional to the user call arrival rate $\lambda_{c}$ and the number of calls originated and terminated by the user, $N$, for updating the UPLs. Thus, the average cost per time unit of maintain the UPL is

$$
M=\left(\lambda_{c} / N\right) C_{m}(k)
$$

Clearly, if a user neither calls nor receives calls, the UPLs should not be changed. If the user makes many calls, the system should be prepared to update the list every $\mathrm{M}$ calls, if necessary.

\section{INTELLIGENT NETWORK SERVICE IN UMTS/IMT-2000}

\subsection{Location update protocol}

Figures $2 \mathrm{a}$ and $2 \mathrm{~b}$ show the conceptual location registration in IMT$2000 /$ UMTS $[7,8]$, further on referred to as a reference scheme, and in Lee's scheme [1].

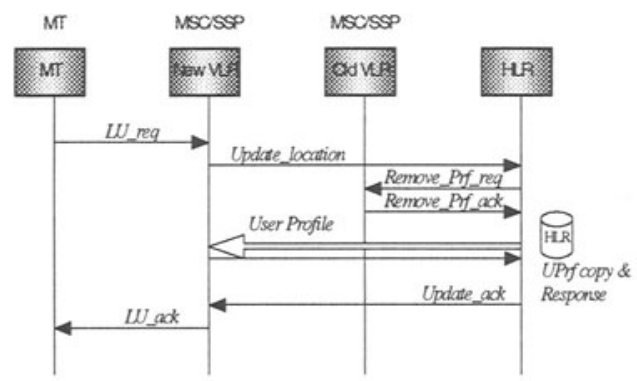

(a) Peference Scherne

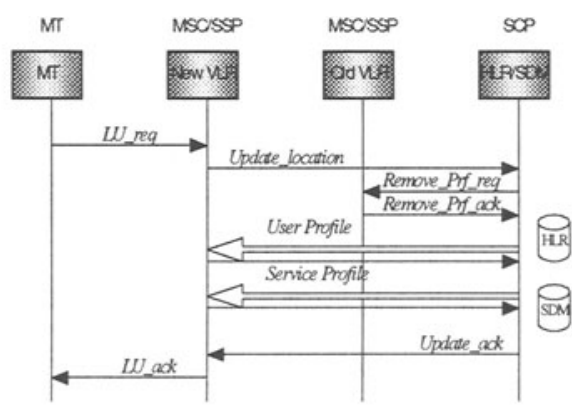

(b) Lee's Scherne

Figure 2. Location update procedures

If an mobile terminal (MT) roams to another mobile switching center (MSC) but still remains inside $\left\{A_{i}\right\}$, the URP scheme does not generate an location update request ( $L U \_r e q$ ) and hence does not involve costs (case 1 in Figure 3). The MT notifies the system only when it leaves the area covered 
by $\left\{A_{i}\right\}$. For the first registration outside $\left\{A_{i}\right\}$ (case 2 in Figure 3 ), the new MSC gets the user profile and the service profile from its MFLA, whereby the new location of the MT is forwarded to the MFLA by a forwarded pointer. If the MT moves to another MSC outside of $\left\{A_{i}\right\}$, the message sequences are identical to the case 2 procedures except for a request for a redirection pointer from the old MSC to the new one, as can be seen in case 3 of Figure 3. The redirection pointer in the location database of the old MSC helps to reduce the miss rate during the call delivery procedure. When an MT re-enters its $\left\{A_{i}\right\}$, the above-mentioned messages are exchanged and in addition the MFLA is notified that the MT is inside $\left\{A_{i}\right\}$ and the forwarded pointer is deleted as shown in case 4 of Figure 3 . In this case, all profiles related to the user still remain in $\left\{A_{i}\right\}$, thus the copy of those profile is unnecessary. This scheme reduces the access to the HLR/SCP as much as possible. The HLR/SCP keeps all the user profiles, service profiles and their pointed MFLAs, which will be updated and maintained offline periodically depending on the number of calls originated and terminated by the users.

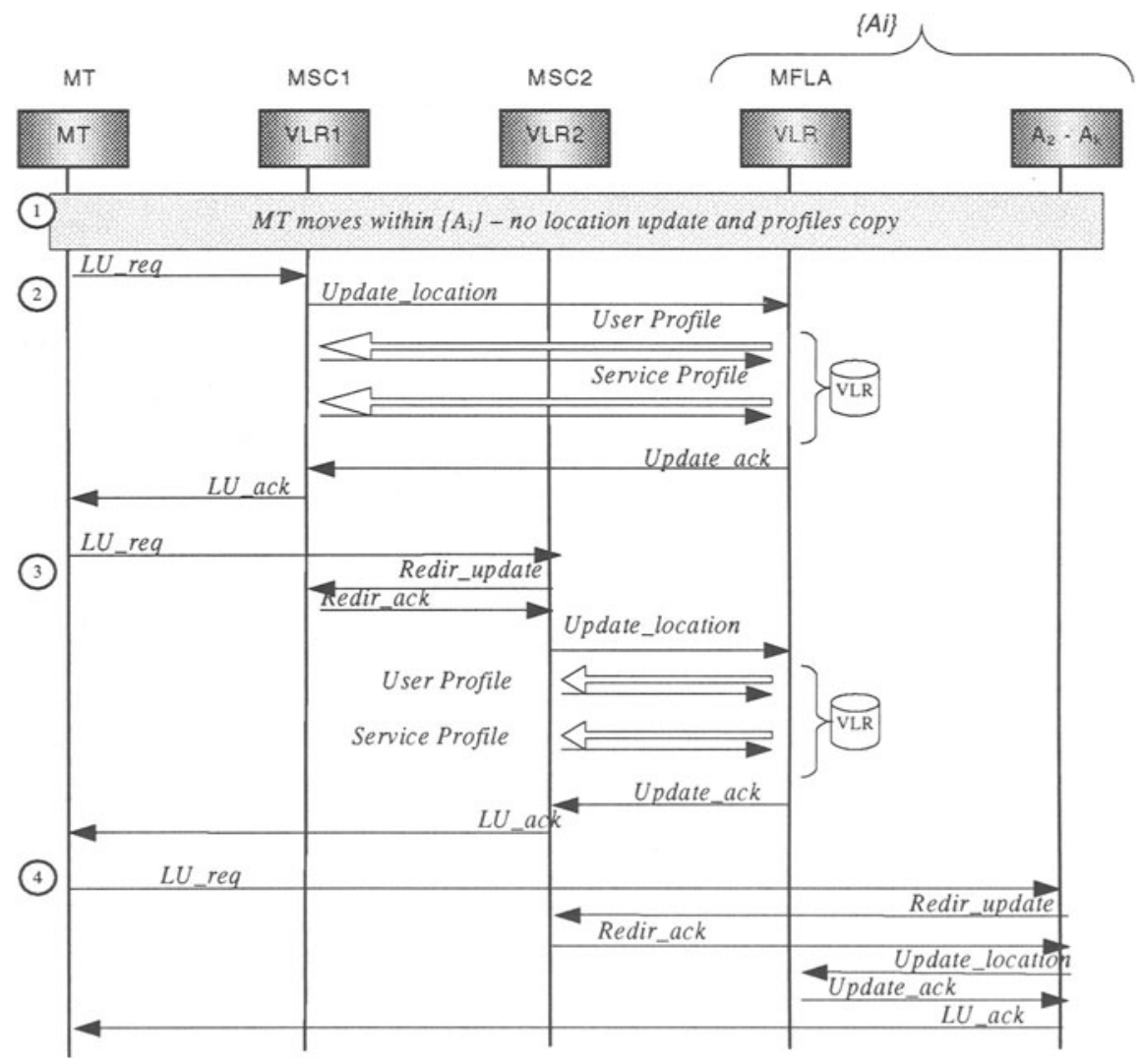

Figure 3. Location update procedures for UPR scheme 


\subsection{IN service call setup}

Figure 4a illustrates a scenario for an IN service call setup for the reference scheme. The call setup request is first routed to the nearest MSC/SSP. The SSP recognizes the IN call and triggers an IN user checkpoint service, which is responsible to check the calling user's right at the SCP. The SCP processes the IN service profile and returns the result to the SSP. Many requests from many MSC/SSPs may result in an overload at the SCP as well as a bottleneck at the HLR.

In the Lee's and UPR schemes, both user profile and service profile are downloaded into the VLR/SSP when the user performs the location registration. Thus, the processes for the $\mathbb{I N}$ service and queries of related profiles take place at the local VLR within the SSP in a cost resulting reduction. The IN service call setup procedure is shown in Figure $4 \mathrm{~b}$.

In this procedure, we assume that the SSP is responsible for chaining the elementary services and applies its basic triggering capabilities of the IN CS$3[2,4]$, which enables two or more services to be active during the call on the same SSP.

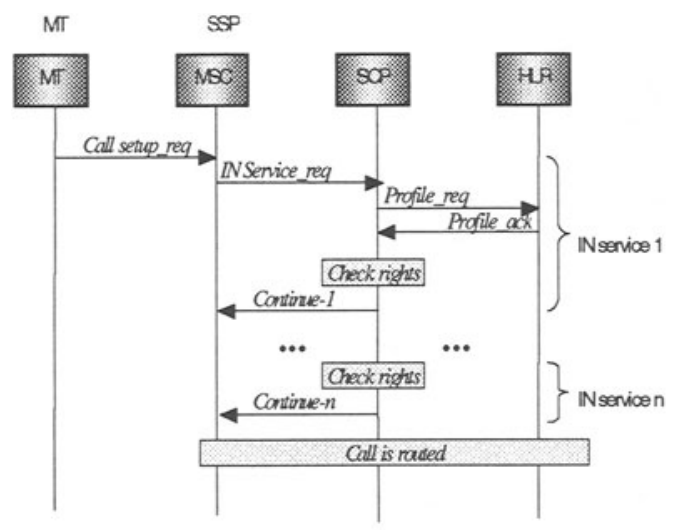

(a) Peference scherne

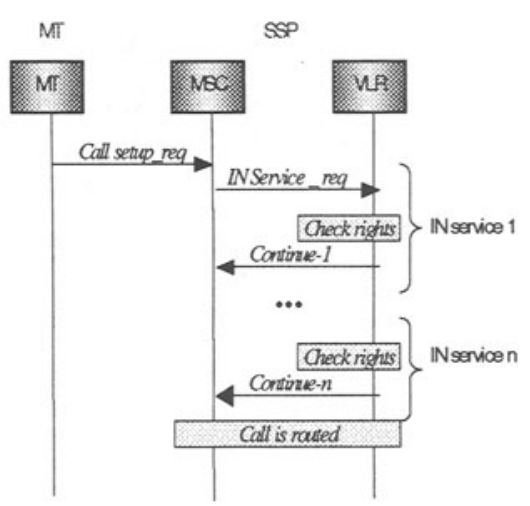

(b) Lœ\&UPR

Figure 4. Scenario for IN service call setup 


\section{PERFORMANCE ANALYSIS}

\subsection{System Analysis}

In this study, we assume that the density of users is uniform throughout the area, that the direction of motion with respect to the border is uniform on $[0,2 \pi)$, and that all the cells are of the same shape and size as well as that they form together a contiguous area. Let $t_{c}$ and $t_{m}$ be independent and identically distributed random variables representing the call inter-arrival time and the location area (LA) residence time. We assume $t_{c}$ and $t_{m}$ to be exponentially distributed with the rate of $\lambda_{c}$ and $\lambda_{m}$, respectively. To simplify the analysis, we assume that all MTs have same values for $\kappa, \lambda_{c}$, and $\lambda_{m}$.

We assume that every VLR serves a location area (LA) that can be connected to a corresponding MSC/SSP. For simplicity, we also make in this contribution the assumption that all VLRs are connected to only one SCP/HLR through a common channel-signaling network No. 7 (SS7 network). Let $p_{I N}$ be the probability that call is an IN service call.

In the UPR with the MFLA strategy, the system maintains a record of each user's most itineraries. Thus we can assume that the probability distribution of a user's location is known exactly. In practice, it will be necessary either to ask the user to provide it or to estimate this using information such as the user's past calling history. The MT keeps track of the list of the location areas $\left\{A_{i}\right\}$. When the system changes the contents of the set, it will notify the MT.

\subsection{Cost Analysis}

To compare the different schemes in terms of cost, each activity is given a cost. The frequency of occurrence for each of these events for the different schemes is computed from the message sequence diagrams. The IN service management cost is divided into two components: (1) the updating cost, $U$, which incurs in completing the location update procedure and service profile download cost, (2) the IN call processing cost, I, which incurs in completing the IN call setup procedure. We consider communication (routing, sending, receiving messages) and database processing as our basic measures of cost. The location update and IN call setup procedures involve the setup of dedicated channels, thus we ignore this cost for all calculations.

Some costs and parameters used to evaluate the analytic model are summarized in Table 1. 
Table 1. List of cost parameters

\begin{tabular}{|c|l|}
\hline Parameters & \multicolumn{1}{c|}{ Meaning } \\
\hline$D_{h}$ & The cost for a query or an update of the HLR \\
\hline$D_{v}$ & The cost for a query or an update of the VLR \\
\hline$D_{s}$ & The cost for a query or an update of the SDM/SCP \\
\hline$N_{l o}$ & The cost for a signaling message through the local link within the MSC \\
\hline$N_{r e}$ & The cost for a signaling message through the remote link to another MSC \\
\hline
\end{tabular}

Table 2. Updating activities, probabilities and costs

\begin{tabular}{|c|c|c|c|l|}
\hline Scheme & Case & \multicolumn{1}{|c|}{ Description } & Probability & \multicolumn{1}{c|}{ Cost } \\
\hline IMT-2000 & -- & -- & -- & $U_{I M T}=6 N_{r e}+2 D_{v}+D_{h}$ \\
\hline Lee & -- & -- & -- & $U_{\text {Lee }}=8 N_{r e}+3 D_{v}+D_{h}$ \\
\hline \multirow{4}{*}{ UPR } & 1 & Moves inside $\left\{\mathrm{A}_{\mathrm{i}}\right\}$ & $p_{u 1}=\kappa^{2}$ & $U_{1}=0$ \\
\cline { 2 - 5 } & 2 & Exit from $\left\{\mathrm{A}_{\mathrm{i}}\right\}$ & $p_{u 2}=\kappa(1-\kappa)$ & $U_{2}=6 N_{r e}+3 D_{v}$ \\
\cline { 2 - 5 } & 3 & Moves outside $\left\{\mathrm{A}_{\mathrm{i}}\right\}$ & $p_{u 3}=(1-\kappa)^{2}$ & $U_{3}=8 N_{r e}+4 D_{v}$ \\
\cline { 2 - 5 } & 4 & Entry into $\left\{\mathrm{A}_{\mathrm{i}}\right\}$ & $p_{u 4}=\kappa(1-\kappa)$ & $U_{4}=4 N_{r e}+2 D_{v}$ \\
\hline
\end{tabular}

Table 3. IN call processing costs

\begin{tabular}{|c|l|}
\hline Scheme & \multicolumn{1}{c|}{ Cost } \\
\hline IMT-2000 & $I_{I M T}=n p_{I N}\left(4 N_{r e}+2 D_{s}+D_{h}\right)$ \\
\hline Lee \& UPR & $I_{\text {Lee }, U P R}=n p_{I N}\left(4 N_{l o}+2 D_{v}\right)$ \\
\hline
\end{tabular}

Tables 2 and 3 summarise all possible events, their corresponding probabilities, and the costs for updating and IN call processing procedures of the reference model, Lee's, and UPR scheme. The average cost per unit time for the UPR scheme can be expressed as the product of the cost and the rate of their occurrence as

$$
U_{U P R}=\lambda_{m} \sum_{i=1}^{4} p_{U i} U_{i}+\frac{\lambda_{c}}{N} C_{m}(k)=\lambda_{m} C_{u}+\frac{\lambda_{c}}{N} C_{m}(k)
$$

The total cost per unit time is the combined cost of location updating and IN service management, denoted as $\mathrm{T}$, which is the sum of the two costs, given as

$$
T=U+I
$$


UPR vs. Reference Scheme

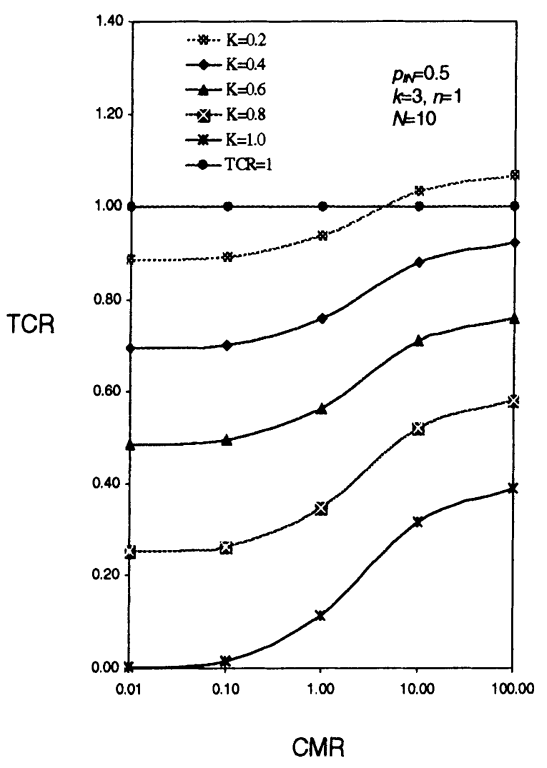

(a)

Lee's vs. Reference Scheme

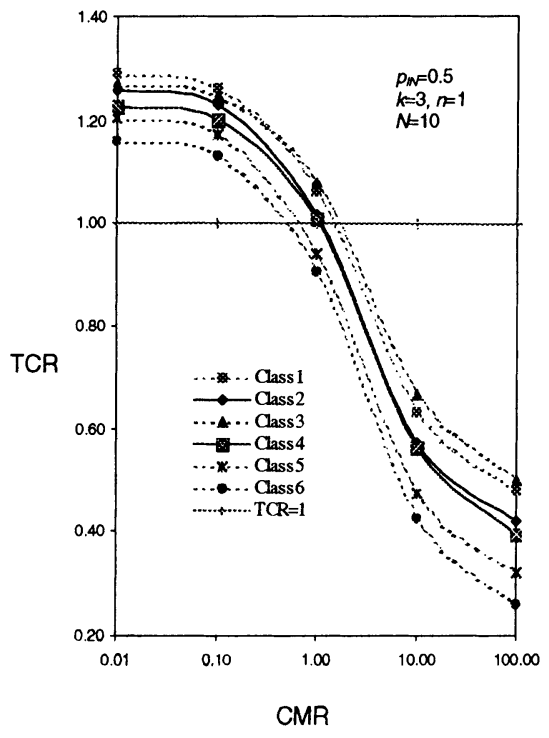

(c)
UPR vs. Reference Scheme

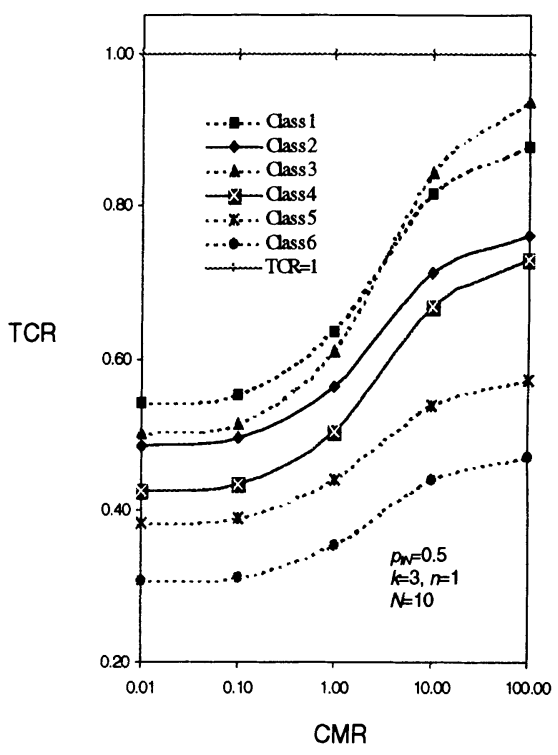

(b)

UPR vs. Leө's Scheme

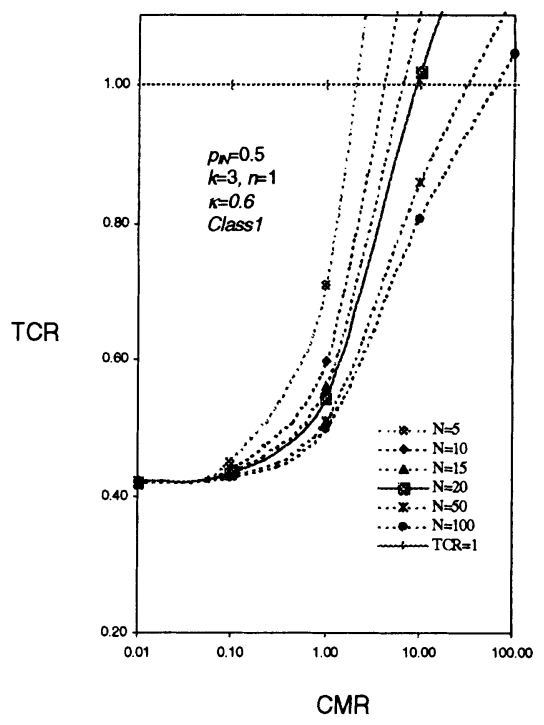

(d)

Figure 5. Total Cost Ratio (TCR) 


\subsection{Numerical Results}

In evaluating and comparing the cost of those strategies, we consider the call to mobility ratio $(C M R)$ as the ratio of the call arrival rate to the mobility rate such as

$$
C M R=\frac{\lambda_{c}}{\lambda_{m}}
$$

In order to be able to estimate the total cost per unit time, the call arrival rate $\lambda_{c}$, and the rate at which the MT moves between LA, $\lambda_{m}$, are needed. We use the call to mobility ratio $(C M R)$ to study the performance of these schemes and compare the cost of those strategies by the ratio of the total costs $(T C R)$ described as follows

$$
T C R=\frac{T}{T_{r e f}}=\frac{C_{u}+C M R^{*} C_{i}}{C_{u-r e f}+C M R^{*} C_{i-r e f}}
$$

Table 4. Coefficient parameters

\begin{tabular}{|l|l|l|l|l|l|}
\hline Class & $\boldsymbol{N}_{l o}$ & $\boldsymbol{N}_{\boldsymbol{r e}}$ & $\boldsymbol{D}_{\boldsymbol{v}}$ & $\boldsymbol{D}_{\boldsymbol{s}}$ & $\boldsymbol{D}_{\boldsymbol{h}}$ \\
\hline 1 & 1 & 2 & 1 & 2 & 3 \\
\hline 2 & 1 & 2 & 1 & 2 & 5 \\
\hline 3 & 1 & 3 & 3 & 2 & 9 \\
\hline 4 & 1 & 3 & 3 & 2 & 15 \\
\hline 5 & 1 & 2 & 1 & 2 & 10 \\
\hline 6 & 1 & 3 & 3 & 2 & 30 \\
\hline
\end{tabular}

The methodology of evaluation used is to establish a common unit of measure for all costs terms, for example time delay. We use the six sets of values for the cost parameters $N_{l o}, N_{r e}, D_{v}, D_{s}$, and $D_{h}$ as shown in Table 4. The value of $N_{l o}$ is normalized to one since it can be seen as the lowest among the other costs. The optimum value of $\mathrm{k}$, the number of location areas where user profiles are replicated, is limited by the incremental cost of maintaining the list. It could be chosen as large as the cost of the list maintaining permits. According to [9], $k \in\{3,4,5\}$ are good choices for the practical systems, to meet the call set-up delay requirements. The number of calls that the system offline updates (or maintains) the user profile lists (UPLs), denoted by $N$, can be chosen as 10 . The cost for updating and maintaining UPLs per user is supposed to be the product of $k$ and its average updating cost $C_{u}$. The number of service checkpoints, $n$, selected from Figure 4 and Table 3 , is one for the analysis. It's clear that if $n$ is greater than one the cost of IN service management reduces due to the access and processing of other IN services still remaining at the local VLR/SSP. 
Figure 5a plots the variation of the normalized total cost between the UPR and reference model schemes with a wide range of CMR (from 0.01 to $100)$. As $K$ increases, the total cost for UPR falls rapidly. Figure $5 b$ shows the ratio of the total cost between the UPR scheme and the reference scheme for the six classes in Table 4. For the proposed strategy, UPR always performs extremely well compared to the reference scheme. When the cost of querying/updating of the HLR is much higher compared to the VLR, the UPR shows increased performance. Lee's scheme (distributed VLR scheme) reduces the IN service management cost significantly only if the user call to mobility ratio is greater than one $(C M R \geq 1)$ as shown in Figure 5c. Additionally, as the frequency of offline updating the user profile lists (UPLs) decreases ( $\mathrm{N}$ varies from 5 to 100), our proposed scheme of UPR gives a better performance than Lee's scheme (Figure 5d).

\section{CONCLUSION}

In this paper, we propose an integration model of IN in future mobile communication networks and a profile-based replication method to efficiently provide IN service. In the proposed network model, two mechanisms have been applied: the distribution of information of the HLR to VLRs and the user profile replication (UPR) strategy for handling the location management and service provisioning. Detailed procedures for the message sequence for location updating and IN service call setup protocols have been presented. Performance analysis shows that the UPR scheme can give the best solution when the replication of user profiles is optimal if the user has a highly localised mobility pattern. We use the most frequent location area (MFLA) to reduce network signalling traffic and database updating as well as the querying delay during location registration and call delivery procedures. The proposed MFLA introduces a new concept for distributed location database access and service control management dependent on per-user mobility behaviour. The numerical results show that the UPR strategy can significantly reduce the total cost compared to other schemes. This UPR strategy could be a very good candidate for distributed location management of future mobile communication networks. In these strategies, user profiles have been copied to a visited network to improve performance but security, integrity, and confidentially must be considered and maintained. 


\section{ABBREVIATIONS}

$\begin{array}{ll}\text { FE } & \text { : Functional Entity } \\ \text { IN } & \text { Intelligent Network } \\ \text { IN CS } & \text { IN Capabilities Set } \\ \text { IMT-2000 } & \text { :International Mobile Telecommunications 2000 } \\ \text { ISDN } & \text { : Integrated Services Digital Network } \\ \text { HLR/VLR } & \text { : Home/Visitor Location Register } \\ \text { LA } & \text { : Location Area } \\ \text { LU } & \text { :ocation Update } \\ \text { MFLA } & \text { : Most Frequency Location Area } \\ \text { MSC } & \text { : Mobile Switching Center } \\ \text { MT } & \text { : Mobile Terminal } \\ \text { PCS } & \text { :Personal Communication Service } \\ \text { PSTN } & \text { : Public Switched Telephone Network } \\ \text { SCM } & \text { :Service Control Module } \\ \text { SCP } & \text { :Service Control Point } \\ \text { SDM } & \text { :Service Data Module } \\ \text { SS7 } & \text { :Signaling System No.7 } \\ \text { SSP } & \text { : Service Switching Point } \\ \text { UMTS } & \text { :Universal Mobile Telecommunication System } \\ \text { UPL } & \text { : User Profile List } \\ \text { UPR } & \text { : User Profile Replication } \\ \text { VHE } & \text { : Virtual Home Environment }\end{array}$

\section{References}

[1] Y. Lee, H. S. Kim, and J. S. Song, "IN service provision using VLR in IMT2000 network", Computer Communications, Vol. 23, No.11, June 2000, pp. 1027-1033.

[2] C. Diquelou, "IN CS-3: The first step towards User Profile Management and the VHE concept", Proceedings of ICIN'2000 Conference, Bordeaux, France, Jan. 2000, pp. 145-150.

[3] T. Magedanz, R. Popescu-Zeletin, "Intelligent Networks", ITP Press, 1996.

[4] ITU-T, Recommendations [Q12xx] of Intelligent Network, Sept. 1999.

[5] M. Laitinen, J. Rantala, "Integration of IN services into future GSM network", IEEE Comm. Mag., June 1995, Vol. 33, No. 6, pp. 76-85.

[6] N. Faggion, T. Hua, "PCSs through the evolution of fixed and mobile comms. and the IN concept", IEEE Network, July/Aug. 1998, Vol. 12, No. 4, pp. 11-18.

[7] ITU-T, Draft Recommendation Q.FIF, Sept. 1997.

[8] ETSI, 3G, TS [22.xxx], Documentations of Technical Specification Group Services and System Aspects, 1999.

[9] G. P. Pollini, C-L. I, "A Profile-based Location Strategy and its Performance", IEEE JSAC, Vol.15, No. 8, Oct. 1997, pp. 1415-1424. 